\title{
Grand Challenge at the Frontiers of Synaptic Neuroscience
}

\author{
P. Jesper Sjöström* \\ Department of Medicine, Department of Neurology and Neurosurgery, Centre for Research in Neuroscience, The Research \\ Institute of the McGill University Health Centre, Montreal General Hospital, Montreal, QC, Canada
}

Keywords: synapse, plasticity, learning, memory, methods, techniques, pathology

\section{INTRODUCTION}

The brain does not operate like a typical computer-there is no central processing unit, there are no separate memory banks, and performance degrades gracefully when individual components fail. This is because all brain functioning is distributed across vast networks of neurons, which are connected by synapses that process and store information. To understand the brain, we therefore need to know how synapses work, hence the central importance of the Synaptic Neuroscience research field that this Frontiers Specialty Journal covers.

In 2000, Abbott and Nelson overviewed the field of synaptic plasticity and likened it to the taming of a beast (Abbott and Nelson, 2000). Synaptic plasticity is indeed a particularly daunting task in neuroscience: experiments are slow and challenging, outcomes vary with precise experimental conditions, and interpretations of the results are inherently prone to disagreement, due to experimental as well as theoretical complexities. We are now more than two decades after the publication of Abbott and Nelson's review, we have seen the immense progress since, and we are in a good position to speculate on what is at the Frontiers of Synaptic Neuroscience. Here, in personal view, I have collected a non-exhaustive list of challenges, phrased as questions.

\section{HOW DOES ONE BUILD A SYNAPSE?}

The key building blocks of the active zone (Südhof, 2012) and the postsynaptic density (Sheng and Kim, 2011) of central synapses are well described. Even so, we may have only barely scratched the surface. Particularly intriguing are the scenarios where the target cell type determines presynaptic release mechanisms, such as in the case of Elfn1 (Stachniak et al., 2019), which-given the diversity of cell types in the brain-implies a potentially enormous diversity of synaptic building blocks across different synapse types. This question is furthermore tightly linked to a related question: How are synapse built during development?

Edited and reviewed by: Idan Segev, Hebrew University of Jerusalem, Israel

*Correspondence: P. Jesper Sjöström jesper.sjostrom@mcgill.ca

Received: 28 July 2021 Accepted: 22 September 2021 Published: 20 October 2021

Citation:

Sjöström PJ (2021) Grand Challenge at the Frontiers of Synaptic Neuroscience.

Front. Synaptic Neurosci. 13:748937. doi: 10.3389/fnsyn.2021.748937

\section{HOW CAN WE MAP PLASTICITY AT ALL SYNAPSE TYPES?}

It has long been known that short-term plasticity at connections made by the same axon can vary dramatically depending on the target neuron type (e.g., Markram et al., 1998). Since the postsynaptic cell determines presynaptic release, this is really a matter of synapse-type rather than cell-type specificity (Blackman et al., 2013). This synapse-type specificity also holds true for long-term plasticity-both phenomenology and mechanisms can vary dramatically depending on the form of synapse at hand (Toth and McBain, 2000; Larsen and Sjöström, 2015). Since there is a plethora of cell types in the brain, there may therefore be a corresponding multiplicity of synapse-type-specific learning rules. Today, the state-of-the-art approach for achieving synapsetype-specific experiments-where both the pre- and the postsynaptic cell identities are knownis still the paired-recording technique. This technique, however, is painstaking and challenging. To create a relatively complete library of synaptic learning rules across all brain regions-the plasticitome, the learning rule equivalent to the genome or the connectome-we clearly need novel 
approaches that have ten to a hundred-fold higher throughput than the paired recording technique. Solving the plasticitome will probably require combining multiple techniques, including electrode arrays (Field et al., 2020), multiple simultaneous wholecell recordings (Perin and Markram, 2013; Lalanne et al., 2016), patch robots (Annecchino et al., 2017; Suk et al., 2017), and optogenetics (Zhang and Oertner, 2007; Emiliani et al., 2015).

\section{IS THERE A GRAND UNIFYING THEORY OF SYNAPTIC LEARNING RULES?}

Many studies have tried to establish if calcium alone can account for synaptic plasticity (Shouval et al., 2002; Rackham et al., 2010; Chindemi et al., 2020), but given the involvement of multiple mechanisms based on dendrite biophysics (Froemke et al., 2010), presynaptic NMDA receptors (Wong et al., 2021) or NMDA receptors that signal unconventionally (Dore et al., 2017), metabotropic glutamate receptors (Nevian and Sakmann, 2006), and learning mechanisms residing in a third cell such as astrocytes (Min and Nevian, 2012; Adamsky et al., 2018), can we really hope to find one calcium model that fits all synapse types? If the calcium hypothesis in synaptic plasticity is not valid for all synapse types, then can we at least establish a phenomenological model that captures plasticity at all synapses? Perhaps it is possible to derive a phenomenological model that can be easily tweaked to account for factors such as synapse position in the dendritic arbor (e.g., Clopath and Gerstner, 2010; Clopath et al., 2010), but whether such an approach is generally feasible for all synapse and cell types remains unknown. Clearly, plasticity at some synapse types does not conform to classical NMDA receptor dependent plasticity, e.g., parallel fibers onto cerebellar Purkinje cells (Hansel et al., 2001), or mossy fibers onto hippocampal CA3 pyramidal cells (Zalutsky and Nicoll, 1990). Also, in a contrasting view to the Grand Unifying Theory, Suvrathan (2018) has convincingly argued that learning rules may in fact be intrinsically heterogenous, to form a basis for learning the behavioral tasks at hand. But perhaps it will be possible to establish a small set of canonical models for a handful of archetypical central synapse classes? Such an effort would help typify synapses of the brain, eventually leading to a robust synapse classification scheme.

\section{HOW DOES THE BRAIN SOLVE THE CREDIT ASSIGNMENT PROBLEM?}

Artificial neural networks have in recent years outperformed humans in several complex tasks. Key to this success is deep learning, i.e., the use of multi-layered networks in combination with the error backpropagation algorithm. This algorithm is used during a separate training phase to assign credit to each synapse, so that the network output error travels backwards to tweak synaptic strengths individually. The brain is clearly a very deep network, so how does it solve the credit assignment problem? Presumably, information cannot travel backwards across synapses in the brain. Storing and using information are furthermore not separate modes of operation for the real brain; your brain is online and functional as you learn new things.

Recent theoretical studies have offered exciting new solutions to these problems. For example, Payeur et al. (2021) recently proposed that by combining short-term plasticity, local dendritic regenerative events, and longterm plasticity in feedback pathways, deep learning can be effectively mimicked by spike burst-dependent plasticity. However, most theoretical propositions similar to this one remain to be validated experimentally (Richards and Lillicrap, 2018; Richards et al., 2019), which can be technically quite challenging.

Because of issues such as the credit assignment problem, it is not clear that the classical forms of cellular learning that dominate the present literature-e.g., long-term potentiation (LTP; Malenka and Bear, 2004) and spiketiming-dependent plasticity (STDP; Markram et al., 2012)-are enough to account for information storage in the brain. As a consequence, demonstrating how the brain solves the credit assignment problem may also tell us which type of synaptic learning rules discovered in vitro are actually relevant in the intact brain, which has been a long-standing debate (Lisman and Spruston, 2005, 2010).

Having said that, it is also important to remember that the question "How does the brain solve the credit assignment problem?" is potentially misleading, because the answer might be: it doesn't. The best biological and technological solutions need not be identical; planes do not flap their wings like birds do.

\section{DOES LONG-TERM SYNAPTIC PLASTICITY UNDERLIE LEARNING AND MEMORY?}

In a short review, Stevens (1998) asked what he called the milliondollar question: Does LTP = memory? He outlined four essential experiments that should be carried out to demonstrate that LTP underlies behavioral learning and memory: blocking LTP prevents behavioral learning; synapses exhibit LTP; behavioral learning causes LTP that when blocked abolishes the learning; and inducing LTP causes behavioral learning. Although it has been established that LTP $=$ memory in simpler model systemssuch as for the gill withdrawal reflex in the sea slug Aplysia (Kandel, 2001)-with the Stevens (1998) definition, it actually remains to be demonstrated that $L T P=$ memory in most mammalian learning models. In fact, most studies of longterm plasticity do not explore beyond an hour or two; clearly not enough to establish a direct link with long-term memory formation (Stevens, 1998). Perhaps the closest we currently get to satisfying the four requirements of Stevens (1998) in a single paper can be found in the amygdala fear conditioning study by Nabavi et al. (2014). It may, however, take some time before the same can be achieved for brain regions with more complex 
roles in learning, such as the hippocampus (Tonegawa et al., 2018).

\section{WHAT IS THE SYNAPTIC BASIS OF NEUROPATHOLOGY?}

Malfunction of synapses and of their plasticity can contribute to severe neuropathology, which has led to the notion of a possible synaptic basis of disease (Lüscher and Isaac, 2009). Epilepsy is a prime example of how excessive activity can hijack plasticity in the healthy brain to yield pathology (Cela and Sjöström, 2019). In fact, both the postsynapse (Kasai et al., 2021) and the presynapse (Van Battum et al., 2015; Perrone-Capano et al., 2021) have been shown to contribute to a broad range of brain diseases as diverse as schizophrenia, autism spectrum disorder, Alzheimer's disease, and amyotrophic lateral sclerosis. By considering neuropathologies as synaptic disease, we may increase our chances of finding novel and specific therapies, since different synapse types are often mechanistically distinct. For example, Walia et al. (2021) recently demonstrated that by specific pharmacologic blockade of calcium-permeable but not calcium-impermeable AMPA receptors in the cochlea, it was possible to protect from excitotoxic pathology during acoustic overexposure, without impairing hearing as such. Promising new treatments will thus likely require detailed mechanistic knowledge of the synaptic pathways at hand.

\section{REFERENCES}

Abbott, L. F., and Nelson, S. B. (2000). Synaptic plasticity: taming the beast. Nat. Neurosci. 3, 1178-1183. doi: 10.1038/8 1453

Adamsky, A., Kol, A., Kreisel, T., Doron, A., Ozeri-Engelhard, N., Melcer, T., et al. (2018). Astrocytic activation generates de novo neuronal potentiation and memory enhancement. Cell 174, 59-71. doi: 10.1016/j.cell.2018.0 5.002

Annecchino, L. A., Morris, A. R., Copeland, C. S., Agabi, O. E., Chadderton, P., and Schultz, S. R. (2017). Robotic automation of in vivo two-photon targeted whole-cell patch-clamp electrophysiology. Neuron 95, 1048-1055. doi: 10.1016/j.neuron.2017.08.018

Bassett, D. S., Cullen, K. E., Eickhoff, S. B., Farah, M. J., Goda, Y., Haggard, P., et al. (2020). Reflections on the past two decades of neuroscience. Nat. Rev. Neurosci. 21, 524-534. doi: 10.1038/s41583-020-0363-6

Blackman, A. V., Abrahamsson, T., Costa, R. P., Lalanne, T., and Sjöström, P. J. (2013). Target cell-specific short-term plasticity in local circuits. Front. Synaptic Neurosci. 5:11. doi: 10.3389/fnsyn.2013.00011

Boyden, E. S., Zhang, F., Bamberg, E., Nagel, G., and Deisseroth, K. (2005). Millisecond-timescale, genetically targeted optical control of neural activity. Nat. Neurosci. 8, 1263-1268. doi: 10.1038/nn1525

Cela, E., and Sjöström, P. J. (2019). Novel optogenetic approaches in epilepsy research. Front. Neurosci. 13:947. doi: 10.3389/fnins.2019.00947

Chindemi, G., Abdellah, M., Amsalem, O., Benavides-Piccione, R., Delattre, V., Doron, M., et al. (2020). A calcium-based plasticity model predicts long-term potentiation and depression in the neocortex. bioRxiv 2020.2004.2019.043117. doi: 10.1101/2020.04.19.043117

Clopath, C., Busing, L., Vasilaki, E., and Gerstner, W. (2010). Connectivity reflects coding: a model of voltage-based STDP with homeostasis. Nat. Neurosci. 13, 344-352. doi: 10.1038/nn.2479

Clopath, C., and Gerstner, W. (2010). Voltage and spike timing interact in STDPa unified model. Front. Synaptic Neurosci. 2:12. doi: 10.3389/fnsyn.2010.00025

\section{WHAT WILL THE NEXT BIG TECHNOLOGICAL BREAKTHROUGHS BE?}

There is a broad consensus that major advances in neuroscience are typically driven by new technologies (Bassett et al., 2020). It is for example difficult to underestimate the impact of optogenetics (Boyden et al., 2005) on modern neuroscience. Key to the next big breakthroughs will be the audacity to ask daring and wishful questions, such as: How can we read out all the synaptic weights in live brain tissue? Because what was once science fiction-e.g., the activation of individual neurons with light-is now science.

\section{AUTHOR CONTRIBUTIONS}

The author confirms being the sole contributor of this work and has approved it for publication.

\section{FUNDING}

PJS was funded by FRQS Chercheur-Boursier award 254033.

\section{ACKNOWLEDGMENTS}

Olivier Camiré, Hovy Wong, and Airi Watanabe provided helpful feedback.

Dore, K., Stein, I. S., Brock, J. A., Castillo, P. E., Zito, K., and Sjöström, P. J. (2017). Unconventional NMDA receptor signaling. J. Neurosci. 37, 10800-10807. doi: 10.1523/JNEUROSCI.1825-17.2017

Emiliani, V., Cohen, A. E., Deisseroth, K., and Häusser, M. (2015). Alloptical interrogation of neural circuits. J. Neurosci. 35, 13917-13926. doi: 10.1523/JNEUROSCI.2916-15.2015

Field, R. E., D’Amour, J. A., Tremblay, R., Miehl, C., Rudy, B., Gjorgjieva, J., et al. (2020). Heterosynaptic plasticity determines the set point for cortical excitatory-inhibitory balance. Neuron 106, 842-854. doi: 10.1016/j.neuron.2020.03.002

Froemke, R. C., Letzkus, J. J., Kampa, B. M., Hang, G. B., and Stuart, G. J. (2010). Dendritic synapse location and neocortical spike-timing-dependent plasticity. Front. Synaptic Neurosci. 2:29. doi: 10.3389/fnsyn.2010.00029

Hansel, C., Linden, D. J., and D'Angelo, E. (2001). Beyond parallel fiber LTD: the diversity of synaptic and non-synaptic plasticity in the cerebellum. Nat. Neurosci. 4, 467-475. doi: 10.1038/87419

Kandel, E. R. (2001). The molecular biology of memory storage: a dialogue between genes and synapses. Science 294, 1030-1038. doi: 10.1126/science.1067020

Kasai, H., Ziv, N. E., Okazaki, H., Yagishita, S., and Toyoizumi, T. (2021). Spine dynamics in the brain, mental disorders and artificial neural networks. Nat. Rev. Neurosci. 22, 407-422 doi: 10.1038/s41583-021-00467-3

Lalanne, T., Abrahamsson, T., and Sjöström, P. J. (2016). Using multiple wholecell recordings to study spike-timing-dependent plasticity in acute neocortical slices. CSH Protoc. 6, 573-583. doi: 10.1101/pdb.prot091306

Larsen, R. S., and Sjöström, P. J. (2015). Synapse-type-specific plasticity in local circuits. Curr. Opin. Neurobiol. 35, 127-135. doi: 10.1016/j.conb.2015.08.001

Lisman, J., and Spruston, N. (2005). Postsynaptic depolarization requirements for LTP and LTD: a critique of spike timing-dependent plasticity. Nat. Neurosci. 8, 839-841. doi: 10.1038/nn0705-839

Lisman, J., and Spruston, N. (2010). Questions about STDP as a general model of synaptic plasticity. Front. Synaptic Neurosci. 3:5. doi: 10.3389/fnsyn.2010.00140

Lüscher, C., and Isaac, J. T. (2009). The synapse: center stage for many brain diseases. J. Physiol. 587, 727-729. doi: 10.1113/jphysiol.2008.167742 
Malenka, R. C., and Bear, M. F. (2004). LTP and LTD: an embarrassment of riches. Neuron 44, 5-21. doi: 10.1016/j.neuron.2004.09.012

Markram, H., Gerstner, W., and Sjöström, P. J. (2012). Spike-timingdependent plasticity: a comprehensive overview. Front. Synaptic Neurosci. 4:2. doi: $10.3389 /$ fnsyn.2012.00002

Markram, H., Wang, Y., and Tsodyks, M. (1998). Differential signaling via the same axon of neocortical pyramidal neurons. Proc. Natl. Acad. Sci. USA. 95, 5323-5328. doi: 10.1073/pnas.95.9.5323

Min, R., and Nevian, T. (2012). Astrocyte signaling controls spike timingdependent depression at neocortical synapses. Nat. Neurosci. 15, 746-753. doi: 10.1038/nn.3075

Nabavi, S., Fox, R., Proulx, C. D., Lin, J. Y., Tsien, R. Y., and Malinow, R. (2014). Engineering a memory with LTD and LTP. Nature 511, 348-352. doi: $10.1038 /$ nature13294

Nevian, T., and Sakmann, B. (2006). Spine $\mathrm{Ca}^{2+}$ signaling in spike-timing-dependent plasticity. J. Neurosci. 26, 11001-11013. doi: 10.1523/JNEUROSCI.1749-06.2006

Payeur, A., Guerguiev, J., Zenke, F., Richards, B. A., and Naud, R. (2021). Burst-dependent synaptic plasticity can coordinate learning in hierarchical circuits. Nat. Neurosci. 24, 1010-1019. doi: 10.1038/s41593-02100857-x

Perin, R., and Markram, H. (2013). A computer-assisted multi-electrode patchclamp system. J. Vis. Exp. 80:e50630. doi: 10.3791/50630

Perrone-Capano, C., Volpicelli, F., Penna, E., Chun, J. T., and Crispino, M. (2021). Presynaptic protein synthesis and brain plasticity: from physiology to neuropathology. Prog. Neurobiol. 202:102051. doi: 10.1016/j.pneurobio.2021.102051

Rackham, O., Tsaneva-Atanasova, K., Ganesh, A., and Mellor, J. (2010). A $\mathrm{Ca}^{2+}$-based computational model for NMDA receptor-dependent synaptic plasticity at individual post-synaptic spines in the hippocampus. Front. Synaptic Neurosci. 3:12. doi: 10.3389/fnsyn.2010.00031

Richards, B. A., and Lillicrap, T. P. (2018). Dendritic solutions to the credit assignment problem. Curr. Opin. Neurobiol. 54, 28-36. doi: 10.1016/j.conb.2018.08.003

Richards, B. A., Lillicrap, T. P., Beaudoin, P., Bengio, Y., Bogacz, R., Christensen, A., et al. (2019). A deep learning framework for neuroscience. Nat. Neurosci. 22, 1761-1770. doi: 10.1038/s41593-019-0520-2

Sheng, M., and Kim, E. (2011). The postsynaptic organization of synapses. Cold Spring Harb. Perspect. Biol. 3:a005678. doi: 10.1101/cshperspect. a 005678

Shouval, H. Z., Bear, M. F., and Cooper, L. N. (2002). A unified model of NMDA receptor-dependent bidirectional synaptic plasticity. Proc. Natl. Acad. Sci. USA. 99, 10831-10836. doi: 10.1073/pnas.152343099

Stachniak, T. J., Sylwestrak, E. L., Scheiffele, P., Hall, B. J., and Ghosh, A. (2019). Elfn1-induced constitutive activation of mGluR7 determines frequency-dependent recruitment of somatostatin interneurons. J. Neurosci. 39, 4461-4474. doi: 10.1523/JNEUROSCI.2276-18.2019

Stevens, C. F. (1998). A million dollar question: does LTP = memory? Neuron 20, 1-2. doi: 10.1016/S0896-6273(00)80426-2
Südhof, T. C. (2012). The presynaptic active zone. Neuron 75, 11-25. doi: 10.1016/j.neuron.2012.06.012

Suk, H. J., van Welie, I., Kodandaramaiah, S. B., Allen, B., Forest, C. R., and Boyden, E. S. (2017). Closed-loop real-time imaging enables fully automated cell-targeted patch-clamp neural recording in vivo. Neuron 95, 1037-1047. doi: 10.1016/j.neuron.2017.08.011

Suvrathan, A. (2018). Beyond STDP-towards diverse and functionally relevant plasticity rules. Curr. Opin. Neurobiol. 54, 12-19. doi: 10.1016/j.conb.2018.06.011

Tonegawa, S., Morrissey, M. D., and Kitamura, T. (2018). The role of engram cells in the systems consolidation of memory. Nat. Rev. Neurosci. 19, 485-498. doi: $10.1038 / s 41583-018-0031-2$

Toth, K., and McBain, C. J. (2000). Target-specific expression of pre- and postsynaptic mechanisms. J. Physiol. 525(Pt 1), 41-51. doi: $10.1111 / j .1469-7793.2000 .00041 . x$

Van Battum, E. Y., Brignani, S., and Pasterkamp, R. J. (2015). Axon guidance proteins in neurological disorders. Lancet Neurol. 14, 532-546. doi: 10.1016/S1474-4422(14)70257-1

Walia, A., Lee, C., Hartsock, J., Goodman, S. S., Dolle, R., Salt, A. N., et al. (2021). Reducing auditory nerve excitability by acute antagonism of $\mathrm{Ca}(2+)$-permeable AMPA receptors. Front. Synaptic Neurosci. 13:680621. doi: $10.3389 /$ fnsyn.2021.680621

Wong, H. H., Rannio, S., Jones, V., Thomazeau, A., and Sjöström, P. J. (2021). NMDA receptors in axons: there's no coincidence. J. Physiol. 599, 367-387. doi: $10.1113 /$ JP280059

Zalutsky, R. A., and Nicoll, R. A. (1990). Comparison of two forms of longterm potentiation in single hippocampal neurons. Science 248, 1619-1624. doi: $10.1126 /$ science. 2114039

Zhang, Y. P., and Oertner, T. G. (2007). Optical induction of synaptic plasticity using a light-sensitive channel. Nat. Methods 4, 139-141. doi: $10.1038 /$ nmeth 988

Conflict of Interest: The author declares that the research was conducted in the absence of any commercial or financial relationships that could be construed as a potential conflict of interest.

Publisher's Note: All claims expressed in this article are solely those of the authors and do not necessarily represent those of their affiliated organizations, or those of the publisher, the editors and the reviewers. Any product that may be evaluated in this article, or claim that may be made by its manufacturer, is not guaranteed or endorsed by the publisher.

Copyright $\odot 2021$ Sjöström. This is an open-access article distributed under the terms of the Creative Commons Attribution License (CC BY). The use, distribution or reproduction in other forums is permitted, provided the original author(s) and the copyright owner(s) are credited and that the original publication in this journal is cited, in accordance with accepted academic practice. No use, distribution or reproduction is permitted which does not comply with these terms. 